\title{
Validation of Measured Damping Trends for Flight-Like Vehicle Panel/Equipment Including a Range of Cable Harness Assemblies
}

\author{
Andrew M. Smith, ${ }^{*}$ R. Benjamin Davis, ${ }^{\dagger}$ \\ NASA Marshall Space Flight Center, Huntsville, AL, 35812, USA
}

Bruce T. LaVerde, ${ }^{\ddagger}$

ERC, Inc., Huntsville, AL, 35805, USA

Clay W. Fulcher, ${ }^{\S}$ Douglas C. Jones, ${ }^{\Uparrow}$ James M. Waldon, ${ }^{\circledR}$ and Benjamin B. Craigmyle ${ }^{\S}$

Jacobs Engineering, Huntsville, AL, 35806, USA

\begin{abstract}
This validation study examines the effect on vibroacoustic response resulting from the installation of cable bundles on a curved orthogrid panel. Of interest is the level of damping provided by the installation of the cable bundles and whether this damping could be potentially leveraged in launch vehicle design. The results of this test are compared with baseline acoustic response tests without cables. Damping estimates from the measured response data are made using a new software tool that leverages a finite element model of the panel in conjunction with advanced optimization techniques. While the full test series is not yet complete, the first configuration of cable bundles that was assessed effectively increased the viscous critical damping fraction of the system by as much as 0.02 in certain frequency ranges.
\end{abstract}

\section{Nomenclature}

Acronyms

ET40 Structural Dynamics Test Branch

EV31 Vehicle Loads and Strength Branch

FE(M) Finite element (model)

MSFC Marshall Space Flight Center

NESC NASA Engineering and Safety Center

PSD Power spectral density

\section{Introduction}

7 HE exterior panels of launch vehicles are subject to significant acoustic and aero-fluctuating pressures 1 during lift-off and ascent (see figure 1). Validation and refinement of the approaches used to estimate the vibration environments associated with these panels is of major importance to new vehicle programs and has been identified by the NASA Engineering and Safety Center (NESC) as an area of uncertainty that is worthy of on-going study.

*Aerospace Engineer, Vehicle Loads and Strength Branch, Mail Code EV31, MSFC, AL

$\dagger$ Aerospace Engineer, Structural and Dynamics Analysis Branch, Mail Code ER41, MSFC, AL

${ }^{\ddagger}$ Aerospace Engineer, ERC, Inc., 4901 Corporate Dr NW E Huntsville, AL, AIAA Member

$\S$ Aerospace Engineer, Jacobs Engineering, 1500 Perimeter Parkway, Huntsville, AL, AIAA Member

『Aerospace Engineer, Jacobs Engineering, 1500 Perimeter Parkway, Huntsville, AL 
In December 2010, a series of acoustic ground tests were conducted on a flight-like vehicle panel in test facilities at the NASA Marshall Space Flight Center (MSFC) 12 The objective of these tests was to gain a better understanding of the response characteristics of increasingly flight-like launch vehicle structural panel assemblies. Follow-on tests are now underway to quantify the differences in damping that may be expected when cable harnesses are added to the panel configurations under test. This paper presents a damping identification approach that may be employed to estimate the modal damping of the tested configuration. The damping trends observed from response measurements of similar configurations with and without cable harnesses should allow analysts to make more informed estimates of damping for use in analytical models of vehicle systems.

Unfortunately, due to facility delays, the entire test data series is not available at the time of publication. However, example cases are complete and enough data are available to demonstrate that accounting for the presence of cable harnesses in damping models may be appropriate. In addition, these representative cases afford the opportunity to illustrate the usefulness of the new damping identification approach presented here. The test measurements may also serve as a point of reference for validating different methods of reducing vibroacoustic response.

\section{Test Overview and Configurations}

The flight-like test article used in acoustic ground testing at MSFC is a ribstiffened aluminum orthogrid panel. It has a curved outer mold line that approximately represents a $45^{\circ}$ section comprising $1 / 8^{\text {th }}$ of the cylindrical exterior shell of a launch vehicle. The panel is clamped in a baffled condition separating the reverberant chamber from an anechoic room (i.e., a flight-like condition where the exterior side of the panel is excited by the high energy acoustic field). The panel is subjected to acoustic noise excitation energies closely simulating the liftoff event and roughly approximating ascent flight events. Note that fixing the baffled panel so that it is excited by source room energies on one side resembles in-service loading of the launch vehicle. Accelerometer, microphone, and strain gage instrumentation was used to measure the acoustic field and the response of the panel.

The acoustic field is generated in the MSFC reverberant chamber using conditioned air driven by up to four parallel WAS 3000 Modulators that feed sound into the chamber through a single horn. This acoustic power source is utilized to approximate a diffuse acoustic field in a reverberant chamber. The acoustic field is monitored using an array of microphones in front of the test article. The standard configuration of microphones is to center the microphones in seven sectors one inch in front of the test article. A sketch of the test chamber showing the source and receiver rooms set up with the approximate location of the microphone sensor array is presented as figure 2 .

The aluminum panel has a smooth outer surface with small orthogrid construction on the interior surface. It is approximately 81 inches in height. The outer surface is covered with a spray-on thermal protection system and is described by diameter of 216.5 inches. The arc length of the panel is approximately 85 inches which is equivalent to $1 / 8^{t h}$ of the full cylinder circumference.

Pictures of the outer surface of the panel (reverberant chamber side) and the interior surface of the panel (anechoic chamber side) are shown in figure 3 . The pictures illustrate that the test condition is that of a baffled panel with source energies exciting only the exterior panel surface. This baffled condition required some facility modifications, but achieving this more flight-like pressure field excitation is an important refinement of the test setup. There can be large differences in panel response between a baffled setup and other configurations that place the test article near the middle of the reverberant chamber with source energies affecting both sides of the panel.

Figure 4 shows photos of the test article in two different configurations. Both photos show the test article with a small attached equipment assembly, but figure 4 (a) shows the panel without any cable bundles. This configuration does have several bolted joints which should contribute more damping losses than would be present in a completely bare panel. Figure 4 (b) shows the curved orthogrid panel supporting cable brackets in the top region of the panel, a secondary support system for avionics equipment in the lower region, a flight-like electronics box on that secondary platform, as well as cable harness bundles strung across the 


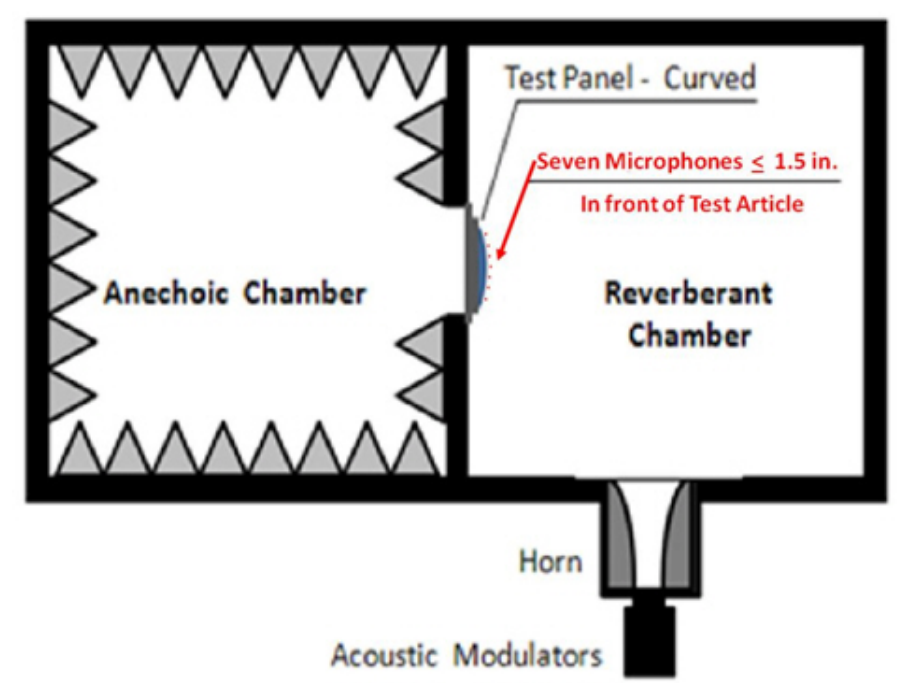

Figure 2. Plan view of test setup showing test article in baffled configuration between the anechoic and reverberant chambers.

bracket system and additional cable bundles extending from the electronics box toward its power and signal sources. The cable bundles extending from the electronics box are attached with flight-like connectors on the box end and then supported by a series of p-clamps across the bottom region of the panel and up the right side. Vibration response transducers - both strain gauges and accelerometers - can also be seen. In Section III test responses measured at various transducers are shown. Figure 4 (b) shows the approximate locations of these transducers with their corresponding numerical designation.

The test series incorporates two cable mounting approaches. It is common in launch vehicles to use brackets with zip ties to permit the routing of multiple cable bundles. It is also common to mount single cable bundles using p-clamps. Both mounting approaches are being explored to assess a range of cable harness configurations. Examples of these various cable mounting systems are shown in figure 5. While complex and difficult to model, each of these mounting arrangements represents an additional source of damping to the system. Section [V] will discuss a new software tool that can help identify the damping associated with the cables without explicitly modeling the complex energy loss mechanisms they introduce to the system.

The test series will consider 56 test cases. To date, only two of those 56 have been assessed. The 56 cases cover 14 configurations each tested at four different acoustic levels. The different configurations are intended to provide insight as to how the quantity of cables affects system damping. The different levels of excitation are meant to assess the nonlinear effects associated with the damping.

\section{Excitation and Response Measurements}

The test setup shown in figure 2 has been used to produce sound pressure levels that are sufficient to elicit flight-like levels of response in the test article system. Figure 6 (a) shows a plot of the acoustic levels recorded by six microphones in the reverberant chamber represented as narrow band pressure spectral density. Figure 6 (b) shows the same acoustic levels represented as a $1 / 3$ octave band averaged sound pressure level spectrum.

By comparing the response of the same transducers it is possible to observe the attenuation achieved through the addition of cable harnesses. Figure 7 presents an overlay of the responses with and without cable harnesses for the six response points shown in figure 4 (b). Attenuation effects are perhaps best observed in figure 7 (c) (transducer 4), which seems to have been sensitive to the presence of the cables over a wide frequency range. It is important to recognize, however, that the observed attenuation is not necessarily purely the result of additional damping introduced by the cable harnesses. When attenuation of response is accompanied by shifts in the frequency of certain response peaks, then the inertial mass effects of the added cables are a likely contributor to the response reduction. If no appreciable shift is observed, one may reason 


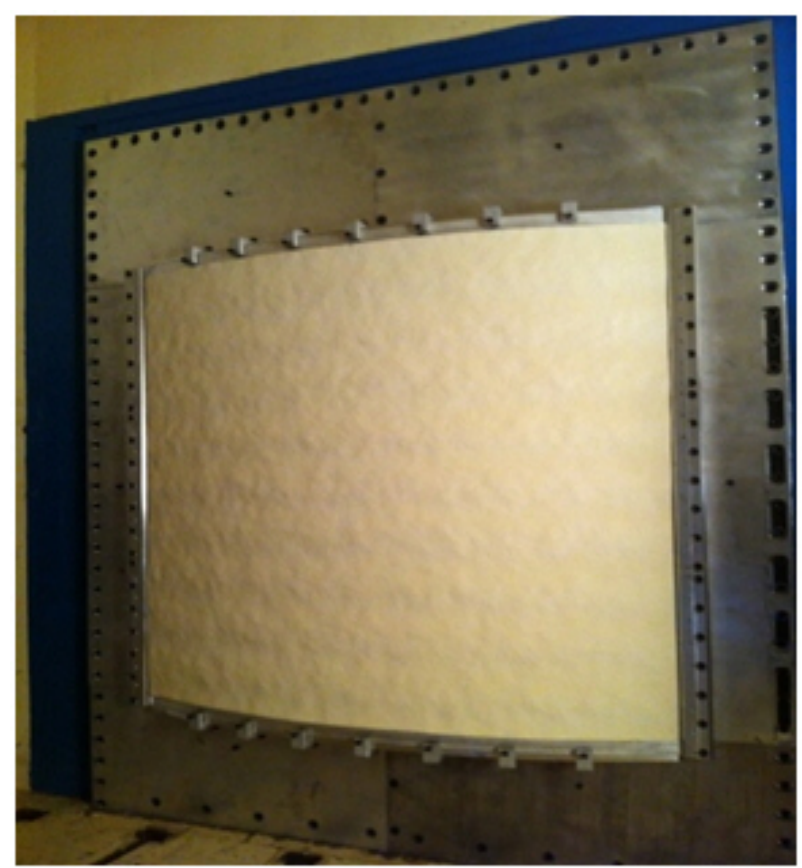

(a)

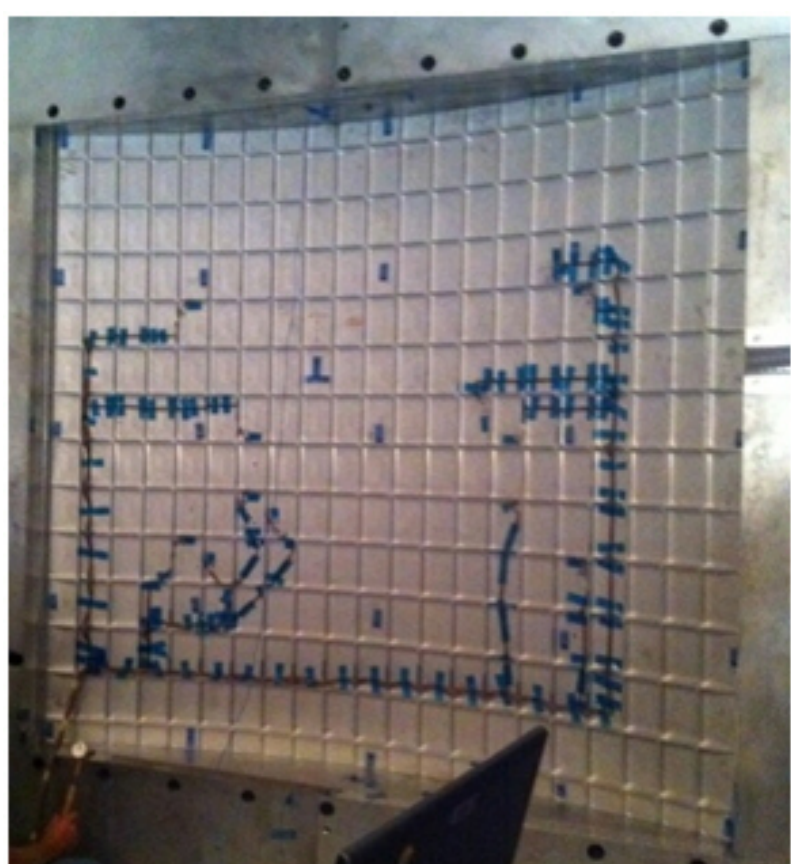

(b)

Figure 3. Reverb-Side Integration of Test Article (left). Anechoic-Side Integration of Test Article at low level of integration. No attached brackets, cable bundles or equipment are included (right).

that additional damping is the root cause of the attenuation.

Figure 7 (a) and (b) represent the response of a pair of transducers in the upper left corner of the panel (i.e., farther away from the cable arrangements). Both provide evidence of attenuation in the range from 100 to $400 \mathrm{~Hz}$. Note these two responses have nearly identical spectral shapes below $600 \mathrm{~Hz}$. In this frequency range the structural bending wavelengths remain large relative to the orthogrid cell size. Above $600 \mathrm{~Hz}$, the responses shown in figure 7 (a) and (b) diverge from each other which is an indication that the bending wavelengths are small enough for the response at the center of an orthogrid cell to be different from the response on the perimeter. Understanding these effects may prove important to explaining the effects that cable bundles have on the response at specific panel locations.

Using standard approach, it is possible to generate a smoothed envelope over the test responses both with and without cables. Such response envelopes are often created for the purposes of component specifications. Since the panel was not integrated with the rest of the vehicle, a $\pm 15 \%$ frequency uncertainty was assumed in both cases. After shifting the response data to account for this uncertainty, smoothed envelopes were drawn over both sets of data. The resulting envelopes corresponding to transducer location 4 are shown in figure 8 . As noted above, this location proved to be particularly sensitive to the presence of cable harnesses in the frequency range between 100 and $400 \mathrm{~Hz}$. As can be seen from figure 8 the observed attenuation in this frequency range due to the presence of the cables is significant. Other locations were not as sensitive to the introduction of cables. Once the test series is complete, a more thorough evaluation will be performed to assess effects that cables have on localized responses.

\section{Identification of Test Damping}

Finite element (FE) models of the test panel with and without attached equipment assemblies were developed. Figure 9 shows the FE model of the panel with the surrounding wall structure. The model contains 60,841 nodes and 57,313 elements. Natural frequencies and modes were calculated in MSC/NASTRAN. Checks were made to ensure the natural frequencies and mode shapes predicted by the model showed good agreement with those found in test. A diffuse acoustic field consistent with the acoustic pressure levels shown in figure 6 was applied to the model. The methodology used to apply the acoustic pressures to the panel can be found in Frady, et al ${ }^{3}$ Since the FE models do not explicitly model the cable harnesses, damping is 


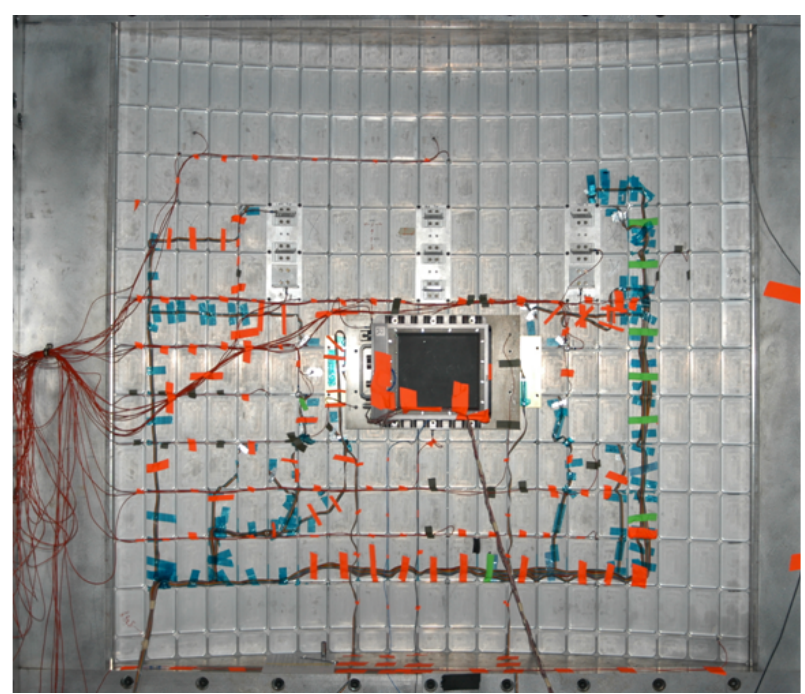

(a)

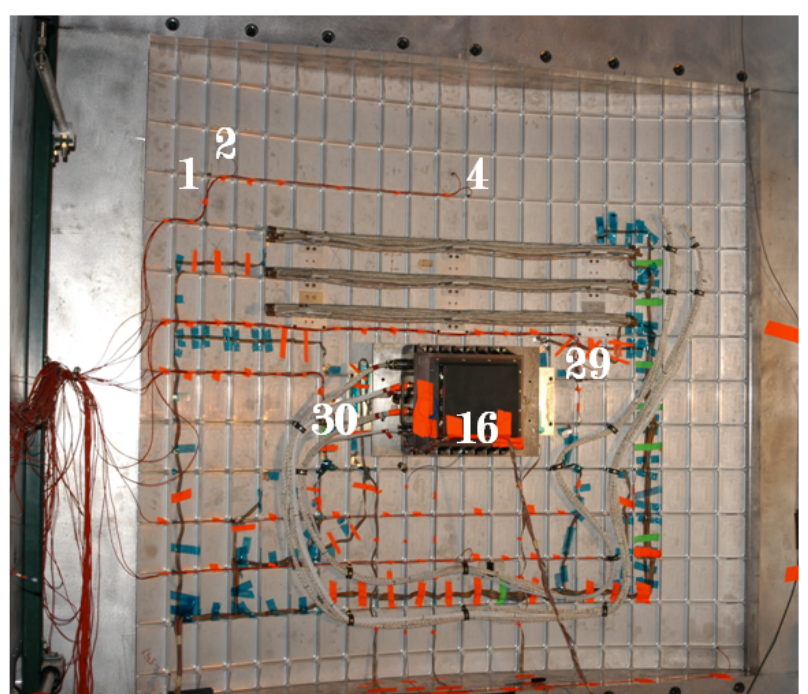

(b)

Figure 4. (a) Anechoic chamber side of the test panel shown with attached mass (a) without cable bundles (b) and with cable bundles. Transducer locations considered in this study are also shown.

the only model parameter that may be adjusted to analytically produce the attenuation effects associated with the presence of the cable harnesses. When a system has too many modes to practically extract modal damping from a test, the analyst is often left to assign damping to an FE model based on little more than engineering judgement. This may result in an iterative process in which the analyst repeatedly adjusts the damping schedule used in the FE analysis with the hope of producing increasingly better agreement with the test data. Such a process can be time consuming and can result in damping schedules that produce sub-optimal agreement with the test data.

To avoid the tedious and time-consuming process of manually assigning damping schedules for use in $\mathrm{FE}$ models, MSFC has developed a new Matlab-based tool which systematically arrives at a damping schedule such that the difference between FE model response and test data is minimized. This tool, called DampID, uses the Matlab Optimization Tool function fmincon to seek the local minimum of a user-defined objective function! In DampID, the components of the objective function consist of statistics associated with the model and test responses. For example, the objective function may be programmed such that the difference between the response peaks found in test and those found by analysis are minimized. Other components of the objective function might include average response, response standard deviation, or response minimum. In each case, DampID calculates these statistics for the FE model response and the test response and then attempts to find a modal damping schedule that will minimize the difference between those two sets of statistics. Weighting coefficients can be applied to favor the agreement between certain statistics over others.

DampID was used in this study to identify the damping observed in the different test configurations. The identified damping schedules are compared in the next section to help elucidate the damping effects associated with the presence of cable harnesses.

\section{Validation using Identified Damping Schedules}

DampID can identify damping schedules based on multiple channels of response data simultaneously. In the cases considered here, the damping schedules were identified based on two response channels: the out-of-plane direction for both transducers 29 and 30. (Refer to figure 4 (b) for the location of these transducers.) Since the responses at these two locations were found to be nearly identical, only the response at the location corresponding to transducer 30 is reported. Figure 10 shows the FE model response overlayed on the test data for this location. Three different test configurations are considered. Figure 10 (a) shows the FE model and test response for the bare panel without any additional mass or cables attached. The solid curve represents the response measured in test while the dashed curve is the response predicted by the $\mathrm{FE}$ analysis. In all cases, the FE analysis uses the damping schedule identified by DampID for the given 




(a)

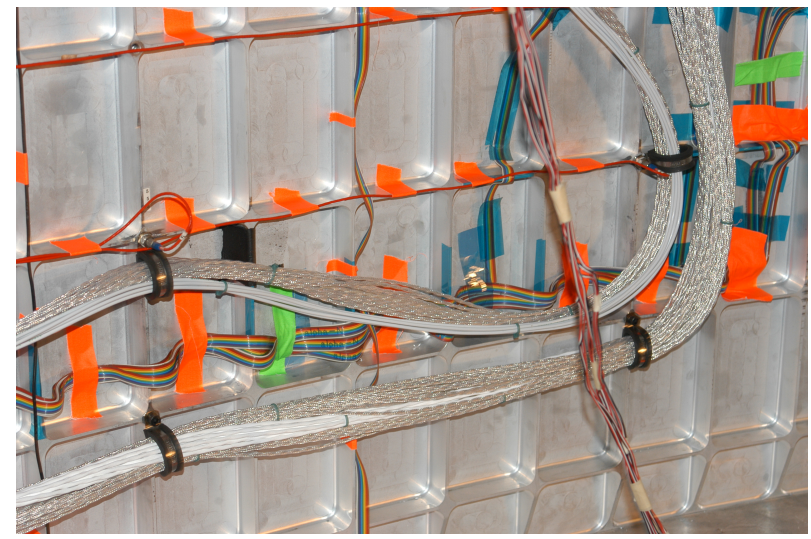

(c)

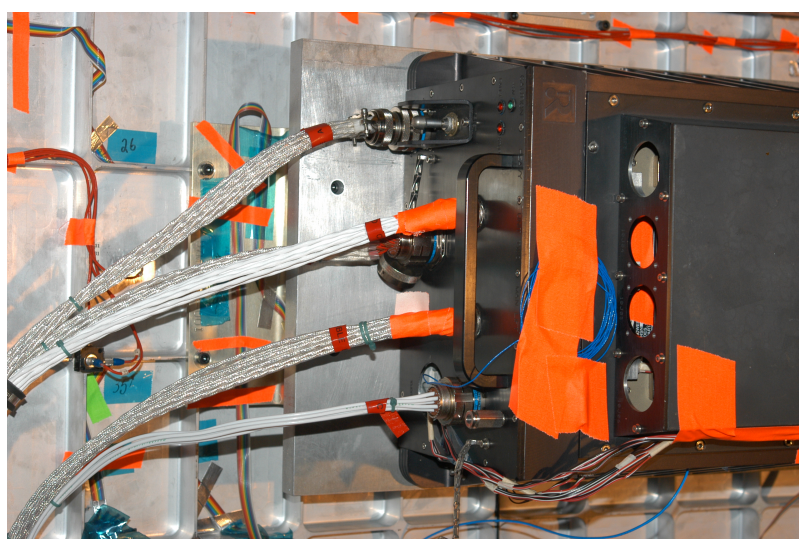

(b)

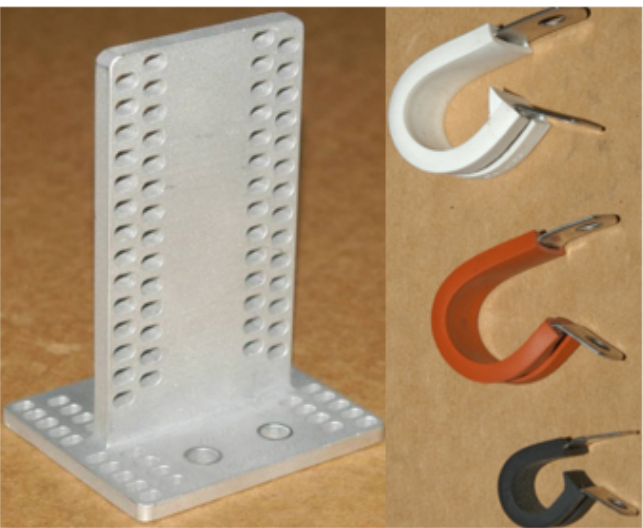

(d)

Figure 5. Examples of potential damping sources associated with cable installation. (a) Cables routed over brackets and secured with plastic tie wraps. (b) Cables attached to flight-like equipment box with pin connectors. (c) Cables routed and secured using p-clamps. (d) Close-up views of a bracket (left) and p-clamps (right).

configuration. Similarly good agreement is seen in figure 10 (b) and (c) where the responses of a mass-loaded panel without cables and with cables are respectively shown. It is instructive to notice how the bare panel response exhibits a different spectral respose than is exhibited by the mass-loaded panels. This is especially true in the low frequency range where the addition of the mass serves to shift the lowest frequency modes even lower. These low frequency modes are more global in nature, so the panel and mass both participate in the motion. Higher frequency modes are more localized, thus explaining why the character of the loaded and unloaded acceleration responses resemble each other in the higher frequency range.

The differences between the bare panel response and the mass-loaded panel responses can also be seen in figure 11 (a) This figure overlays the acceleration PSD responses for the three configurations as they are predicted by the FE analysis. Figure 11 (b) overlays the damping schedules identified by DampID for the three configurations. The overall trend in the three identified damping schedules progresses from the lowest for the unloaded panel to the highest for the mass-loaded panel with attached cables and harness. The damping for the mass-loaded panel without cables falls roughly between these two cases. Also, lower damping corresponds roughly with higher response, especially when comparing the unloaded with loaded panels. These observations tend to confirm the expectations that adding components and cables to the panel will tend to increase damping.

However, several notable exceptions to these general observations can be seen, particularly in a band between 500 and $600 \mathrm{~Hz}$, and for responses higher than $1500 \mathrm{~Hz}$. Between 500 and $600 \mathrm{~Hz}$, DampID identifies a damping of 0.052 for the case without cables, but left damping near 0.02 for the panel with attached cables. This is an exception to the general observation that damping will be higher when cables are present. Several possible causes for this anomaly are under investigation. One possibility is that in this frequency band, transducer 30 shows a lower response without cables than it does with cables (figure 7 (f) confirms that 


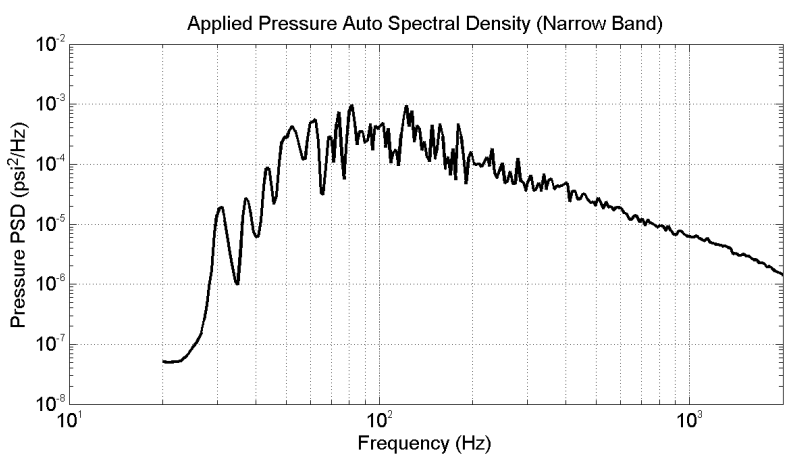

(a)

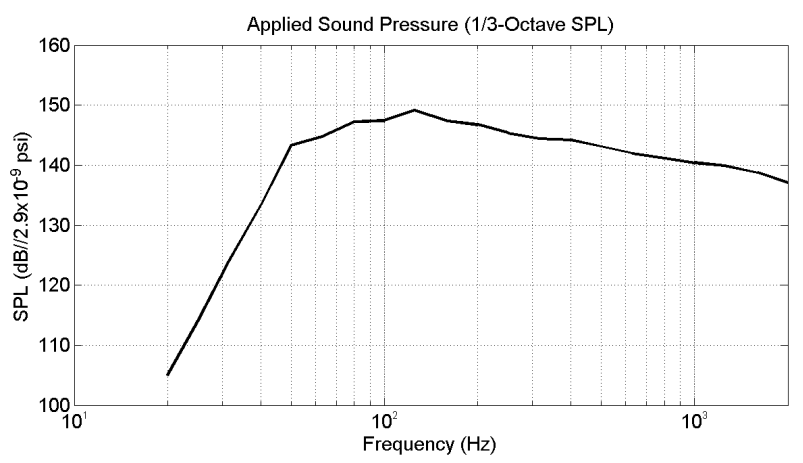

(b)

Figure 6. (a) Measured Narrow band Pressure Spectral Density also applied to models ( $1 / 36^{\text {th }}$ octave band resolution) (b) Same pressure spectrum rendered as Sound Pressure Level $\left(1 / 3^{\text {rd }}\right.$ octave band resolution)

this is in fact the case). In this frequency range, transducer 29 shows the opposite (and expected) trend in which the response with cables is lower than the response without cables. Since the damping schedule was identified using both transducers 29 and 30, any frequency ranges where conflicting responses are exhibited will cause the program to find the best solution for both. The solution for combined channels will not be the best for either channel by itself. If more channels are included in DampID the likelihood of conflicting responses increases. More investigation is needed to explain why the no cable response of transducer 30 is lower than the response of the panel with cables in this frequency range. This discrepancy illustrates why it is important from a practical standpoint to exercise engineering judgement when deciding which channel(s) to use with the DampID.

Above 1,500 Hz, a sharp increase in damping is observed. The reason for this is presently unknown. Possible model-related causes include coarse spatial definition of the pressure and FE mesh density, but upon closer examination these seem to meet the minimum requirements for capturing the structural response. It was observed that the measured test responses, which the optimizer uses to identify damping, showed a dramatic decrease in amplitude similar to that seen in the PSD responses of Figure 7. DampID calculates higher damping in this frequency range to keep the model responses at the measured test levels. A physical understanding of the dramatic decrease in measured structural response after $1,500 \mathrm{~Hz}$ is currently being sought. The issue may also be related to test instrumentation so this possibility is also under scrutiny.

\section{Conclusions and Future Work}

The results of this development test examine the effect of cable bundles on the vibroacoustic response of a mounted panel subject to acoustic excitation. The test data obtained will be used as a point of reference for different methods of reducing vibroacoustic response. While several parts of the test series are still outstanding, it was observed that cables installed on a flight-like panel will lower measured response through an increase in damping. The observed increases in critical damping fraction were as much as 0.02 in certain frequency ranges. However, a more comprehensive examination which considers data from all locations of the panel and various cable configurations needs to be completed before any general conclusions can be made. It is expected that the results of the complete test series will provide the opportunity to reach more definitive conclusions and gain other valuable insights.

In addition to collecting, processing, and interpreting the data from the rest of the test series, future work may include considering external panels there are only lightly mass loaded. These panels tend to be even more responsive than the panels considered here, thus accounting for any additional damping provided by added equipment or cables to these panels will be especially beneficial.

\section{Acknowledgments}

The authors would like to acknowledge the fine support provided by the test organization at MSFC. The dedicated and capable staff of the ET40 department has provided outstanding support to complete an earlier set of ambitious acoustic response tests. They have also been actively developing opportunities to leverage 
the test article and test setup toward valuable follow on tests. The authors would also like to thank our test integrator Mr. Anthony Kelley.

\section{References}

${ }^{1}$ Smith, A., Harrison, P., LaVerde, B., Hunt, R., and Teague, D., "Preliminary Evaluation of Mass Loaded Vehicle Panel Response Estimates and approaches Based on Acoustic Ground Test Results," Proceedings of the Aerospace Testing Seminar, ATS, 2011.

${ }^{2}$ Peck, J., Smith, A., Fulcher, C., LaVerde, B., and Hunt, R., "Development of Component Interface Loads on a Cylindrical Orthogrid Vehicle Section from Test-Correlated Models of a Curved Panel," Proceedings of the Spacecraft and Launch Vehicle Dynamic Environments Workshop, AIAA, 2011.

${ }^{3}$ Frady, G., Duvall, L., Fulcher, C., LaVerde, B., and Hunt, R., "Test-Anchored Vibration Response Predictions For An Acoustically Energized Curved Orthogrid Panel With Mounted Components," Proceedings of JANNAF's 8th Modeling and Simulation Subcommittee (MSS), JANNAF, 2011.

${ }^{4}$ MathWorks, Matlab Optimization Toolkit User Guide, 2011. 


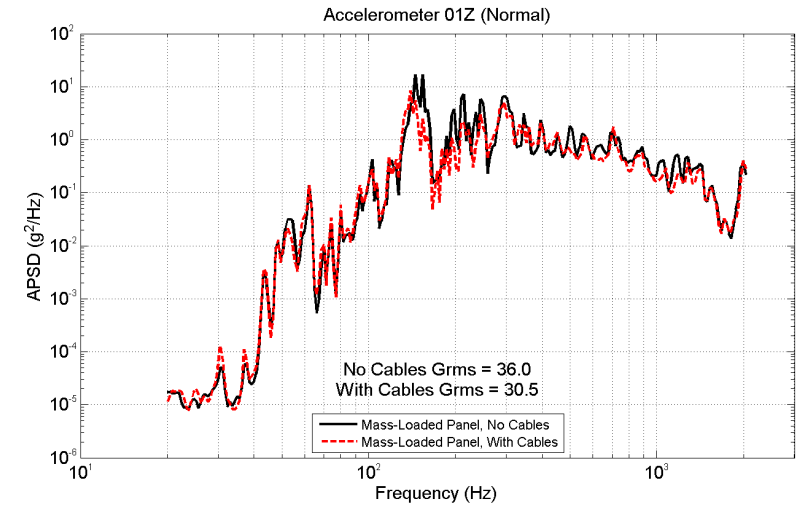

(a)



(c)

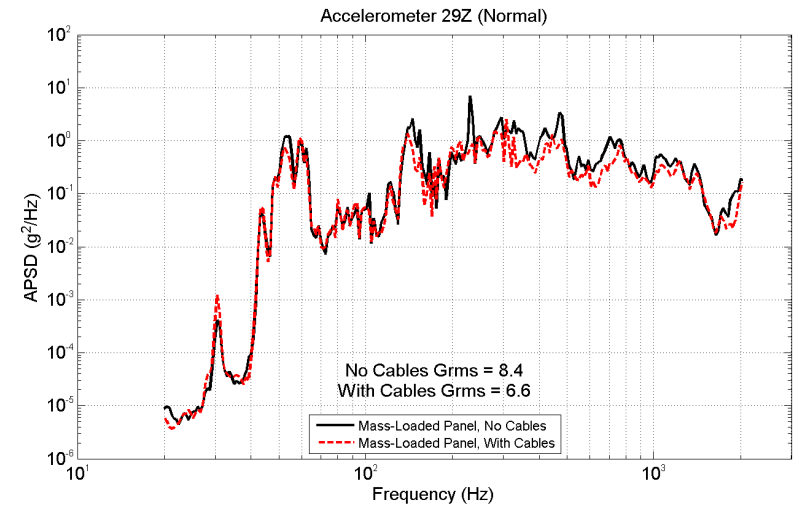

(e)



(b)

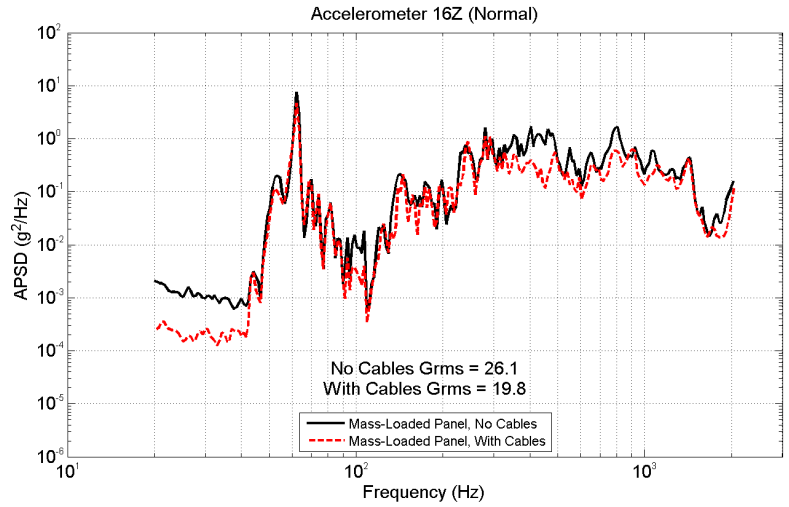

(d)

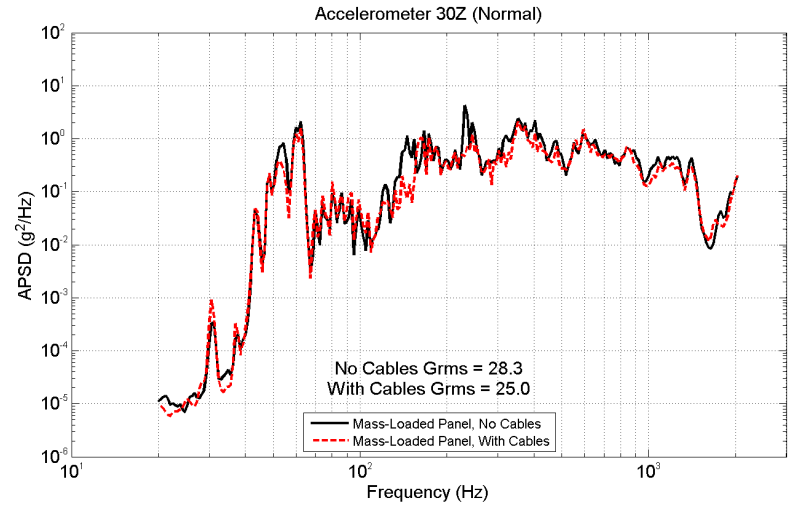

(f)

Figure 7. Test measured acceleration power spectral densities for transducers (a) 01 (b) 02 (c) 04 (d) 16 (e) 29 (f) 30. See figure 4 (b) for the location of these transducers. 


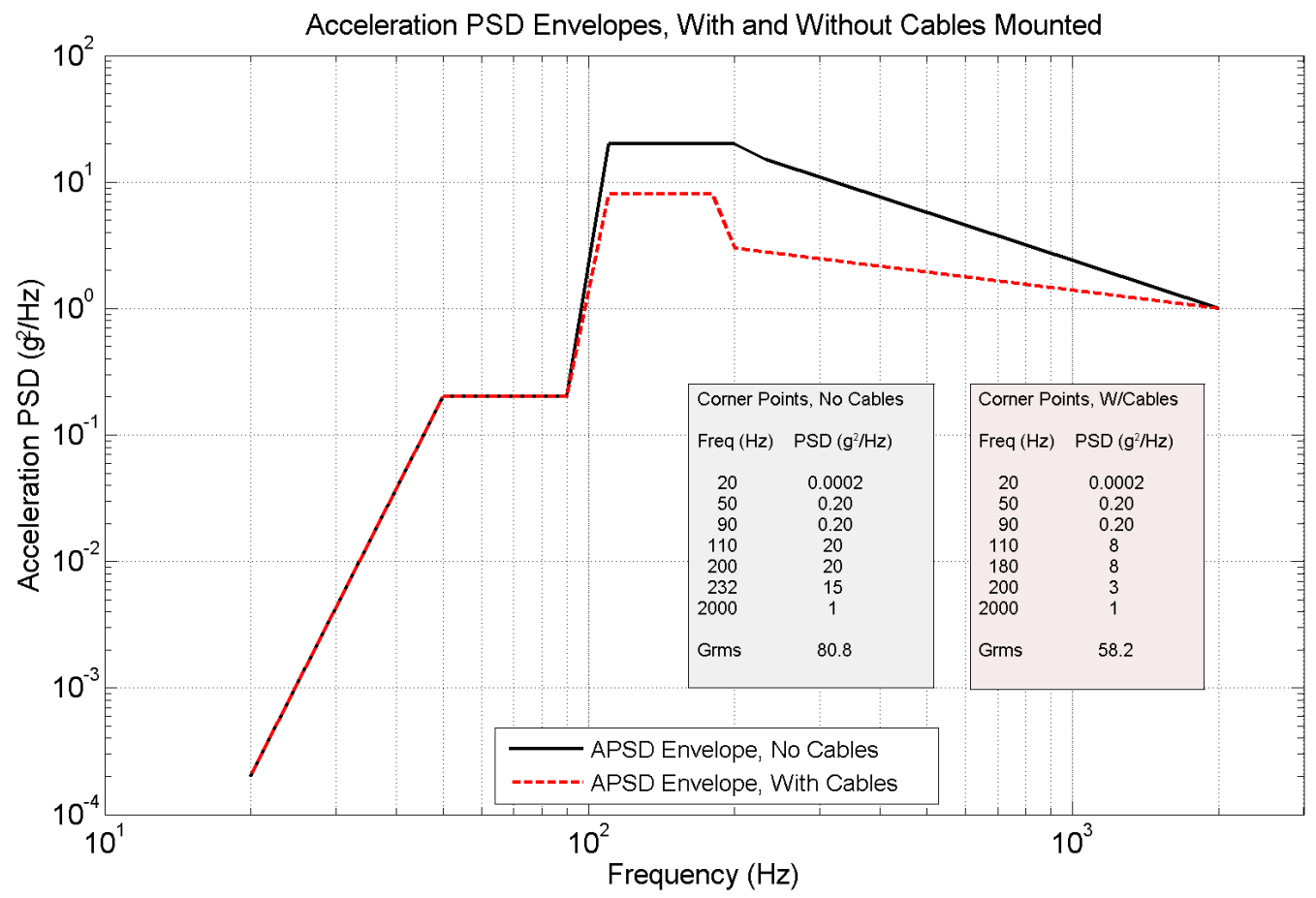

Figure 8. Smoothed envelopes and corner point data corresponding to transducer location 4 for the mass-loaded panel with and without attached cables. 


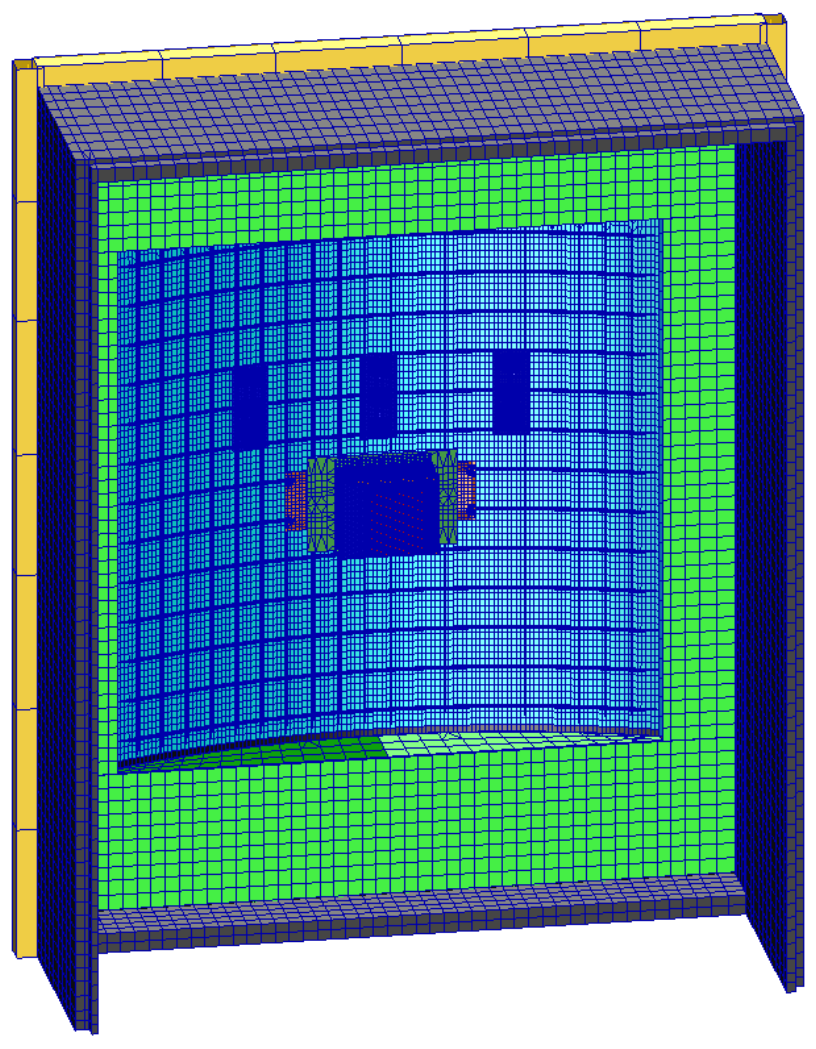

Figure 9. Oblique view of FE model of panel and surrounding support structure. 




(a)

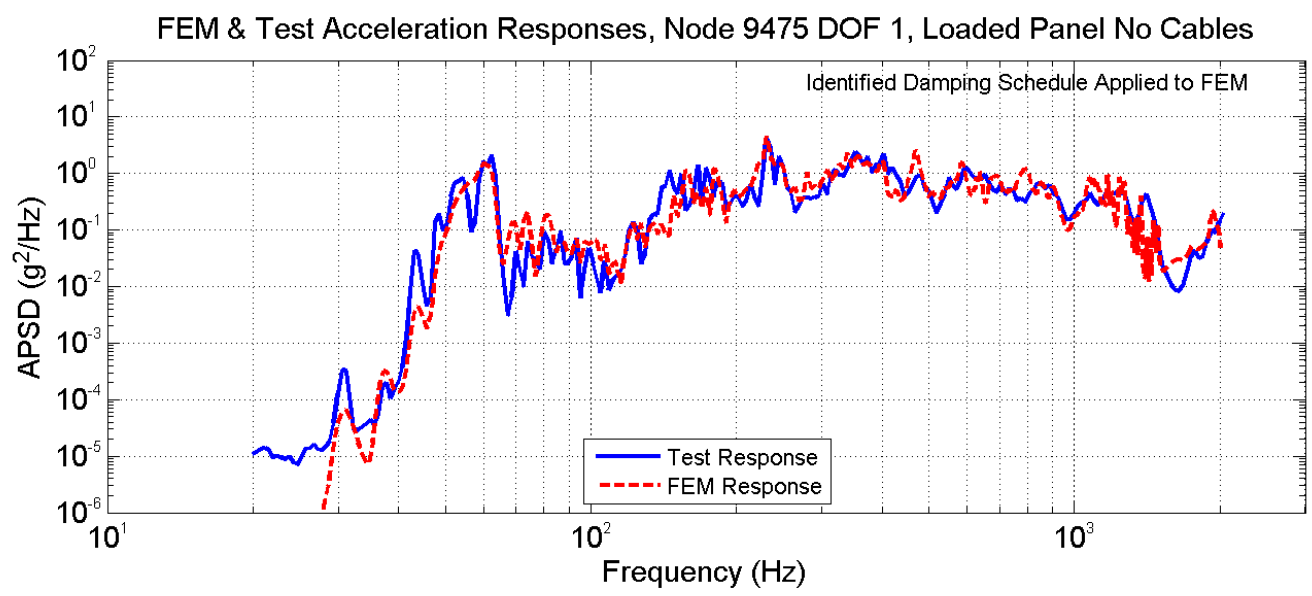

(b)

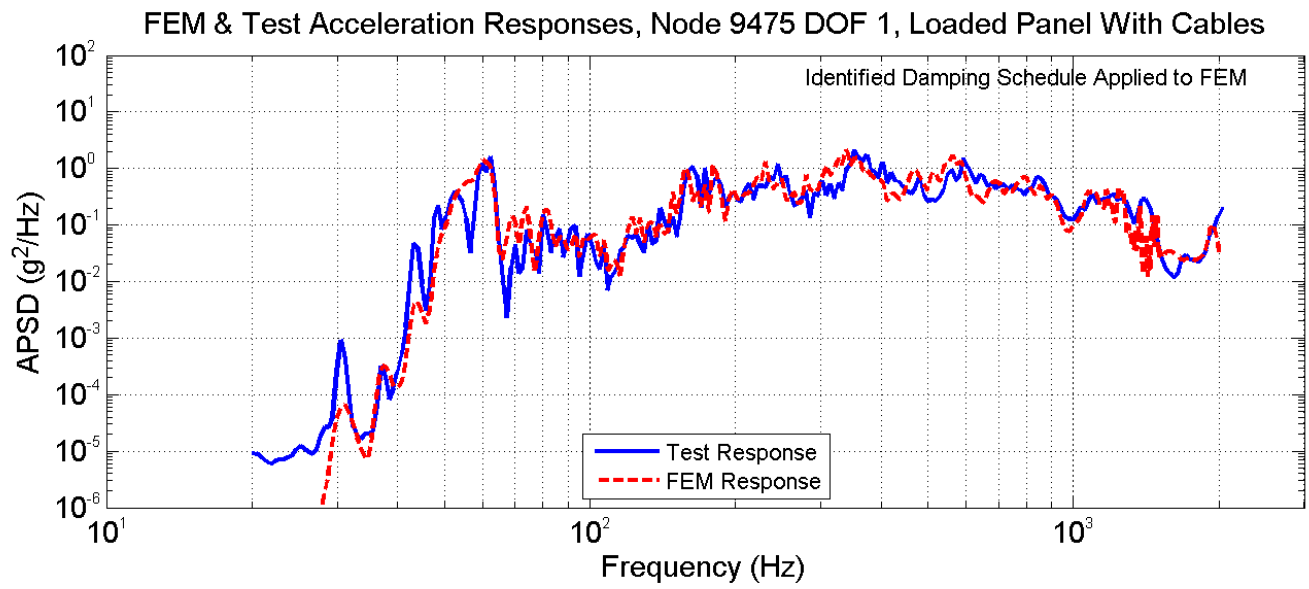

(c)

Figure 10. Finite element model and test acceleration power spectral densities corresponding to (a) a bare panel without attached mass or cables (b) a panel with an attached mass but no cables (c) a panel with an attached mass and attached cables. 


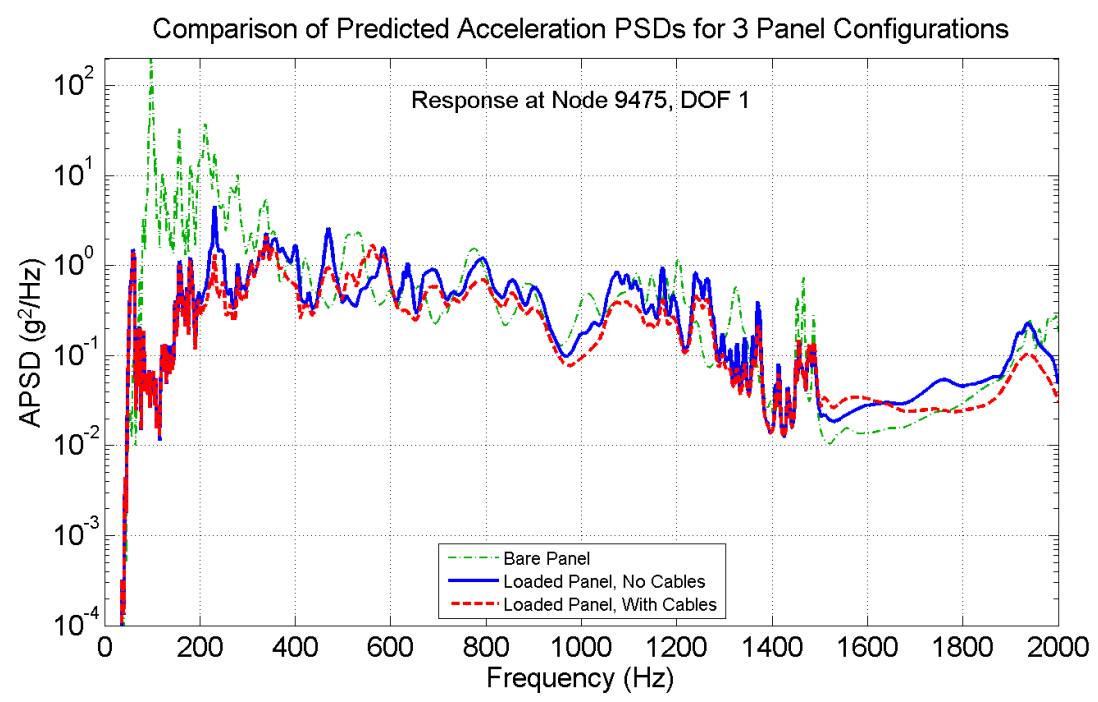

(a)

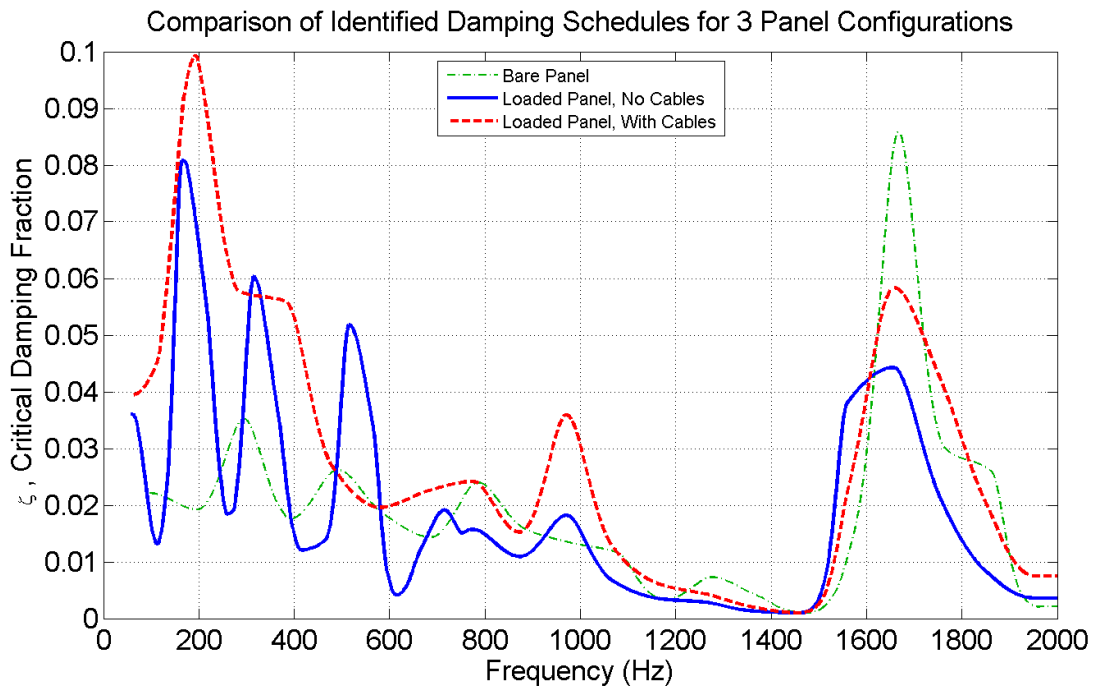

(b)

Figure 11. (a) PSD responses as predicted by FE models for the bare panel, mass-loaded panel without cables and mass-loaded panel with cables. Responses use damping schedules identified for each case. (b) Damping schedules for the three cases as identified by the DampID tool. 